\title{
Searching for alternatively splicing group II introns
}

\author{
Nikolay Kobalo \\ The Laboratory of Computational Problems \\ of Geophysics \\ The Institute of Computational Mathematics \\ and Mathematical Geophysics \\ Novosibirsk, Russia \\ rerf2010rerf@yandex.ru
}

\author{
Denis Vorobyev \\ INSERM U981 \\ Gustave Roussy Cancer Center \\ Villejuif, France
}

\author{
Igor Titov \\ The Laboratory \\ of Molecular-Genetics Systems \\ Cytology and Genetics SB RAS \\ Novosibirsk, Russia \\ titov@bionet.nsc.ru
}

\begin{abstract}
Group II introns are mobile elements present in bacteria, as well as in the eukaryotic organelles. This type of intron is probably the ancestor of nuclear introns. Group II introns have a conservative secondary structure, which determines their ability to self-splice. It is known that some group II introns have the ability to alternative splicing [1]. The possibility of alternative splicing of group II introns is explained by the fact that they can have several different variants of the secondary structure, each of which leads to its own splicing reaction. Based on the introns of group II with the secondary structure described in the literature, we have constructed a generalized model of the structure of introns of this type. In addition, we searched for introns corresponding to our model in the RFAM database and found introns with the possibility of alternative splicing. For the found introns, we performed an analysis of coadaptive nucleotide substitutions in the secondary structure. We also analyzed the effect of alternative splicing on proteins that are the product of the corresponding genes.
\end{abstract}

Keywords - intron group II, secondary structure, alternate splicing

\section{Materials and Methods}

- $\quad$ To build a model of the secondary structure of group II introns, we used a database of known intron structures [2]

- We searched for introns with the possibility of alternative splicing in an open database of bacterial and eukaryotic introns of group II RFAM [3].

- To search for a given secondary structure in genomic sequences, we used the Rscan program [4].

\section{Secondary Structure Models}

The secondary structure of group II introns consists of 5 structural elements called domains. The largest of them is the first domain. At the first stage, for each of the intron domains, except 4, we built several models of the secondary structure. Each model generalized its domain for a group of introns with a similar structure. In addition to the secondary structure itself, described by the positions and lengths of its stems and loops, our model also included:

- Consensus sequences for splicing sites.

- The presence of tertiary interactions of introns. Among them are ebs1 / ibs1, ebs2 / ebs2, $\alpha-\alpha^{\prime}, \beta-\beta{ }^{\prime}, \kappa, \varepsilon$.

In order to evaluate the quality of our models, we calculated the distribution of z-score of the energy for the secondary structure on a random sample of nucleotide sequences. Random sequences had the same dinucleotide composition as real introns. Using the obtained distribution, we set the threshold for the energy of the secondary structure: $\mathrm{z}$ score $<=-1.48$.

We used the constructed models to restore the secondary structure introns from the RFAM database. To do this, we looked for sequences that satisfy our constraints on structures, including constraints on tertiary interactions and the z-score threshold. We also analyzed the protein sequences of the fourth domain of the detected introns for the presence of standard RT motifs in them [5]. The group II introns discovered in this way and their secondary structures were described in [6]. In total, we restored the complete secondary structure of 394 eukaryotic group II introns. Among them, 3 introns of subtype IIB, and the rest IIA. 18 found introns belong to the nuclear genome, 10 to the mitochondrial, 127 to the chloroplast genome and 196 to the plastid genome.

\section{Search for alternative splicing}

Then we used the found boundaries of all domains to detect introns containing several valid splicing variations. Such introns can potentially have the ability to alternative splicing. On the identified candidates, we carried out an analysis of coadaptive nucleotide substitutions in the secondary structure in order to establish the most probable path of their evolution. In addition, for introns located close to each other in the genome, we analyzed their interactions and mutual evolution.

\section{REFERENCES}

[1] G. Eason, B. Noble, and I. N. Sneddon, "On certain integrals of LipsBonnie A. McNeil, Dawn M. Simon and Steven Zimmerly. Alternative splicing of a group II intron in a surface layer protein gene in Clostridium tetani. Nucleic Acids Research, 2014, Vol. 42, No. 3.

[2] Dai, L., Toor, N., Olson, R., Keeping, A., and Zimmerly, S. (2003). Database for mobile group II introns. Nucleic Acids Res. 31: 424-426.

[3] Kalvari I. et al. (2018). Rfam 13.0: shifting to a genome-centric resource for non-coding RNA families. Nucleic Acids Res. 46(D1):D335-D342. DOI: 10.24411/9999-017A-2019-10001 10.1093/nar/gkx1038 8-73.

[4] http://www.softberry.com/freedownloadhelp/rna/rscan/rscan.all.html\#R ScanDescription

[5] Zimmerly, S., Wu, L. (2014). An unexplored diversity of reverse transcriptases in bacteria. Microbiol. Spectr. 3 (2), MDNA3-0058-2014. doi: 10.1128/microbiolspec.MDNA3-0058-2014

[6] Titov I, Kobalo N, Vorobyev D and Kulikov A (2019) A Bioinformatic Method For Identifying Group II Introns In Organella Genomes. Front. Genet. 10:1135. doi: 10.3389/fgene.2019.01135 